\title{
Health care differences across Europe concerning routine IUD Surveillance: Results of the EURAS-IUD study
}

\author{
Pauline De Corte*, Clare Barnett, Thai Do Minh and Klaas Heinemann \\ Center for Epidemiology and Health Research Berlin, Invalidenstraße 115, 10115 Berlin, Germany
}

\begin{abstract}
Objectives: To describe country-specific patterns in routine follow-up visits for newly inserted Intra-Uterine Devices (IUD) and evaluate whether examination frequency is associated with IUD perforation rates and primary healthcare financing.

Study Design: Post-hoc analysis using data from the EURAS-IUD prospective, non-interventional cohort study conducted in 6 European countries from 2010 to 2016

Methods: Women were recruited at the time of IUD insertion. Women with their IUD in situ at 12 months were re-contacted at 5-years and asked about latediagnosed perforations and frequency of IUD position checks. The proportion of women with a check was compared between countries and the healthcare payment models. Perforation incidence was calculated per 1,000 insertions and stratified by country.
\end{abstract}

Results: In countries with a social healthcare payment system, around $80 \%$ of women had a position check and almost all were confirmed with transvaginal ultrasound. In countries with a taxation-based healthcare system, less women were examined (15 to 60\%), however, those having a check were more likely examined through transvaginal ultrasound. Perforation detection did not correspond in analogous manner.

Conclusions: Differences in routine IUD check-up procedures were observed between countries and type of primary healthcare financing. Increased IUD surveillance did not correlate with increased detection of IUD perforations. No obvious correlation between the health payment system and perforation rate could be observed.

\section{Introduction}

Worldwide, $14.3 \%$ of women of reproductive age use an intrauterine device (IUD) as their main contraceptive method [1]. In general, interest in IUDs as a contraceptive method is increasing among women and their health care providers. Today, IUD usage and IUD surveillance varies widely within Europe, with disparities existing both from country to country, as well as within country. Country-level recommendations and policies regarding follow-up (FU) visits following IUD insertion do not align. FU visits are designed to identify any IUD-related problems, confirm the correct IUD position and identify occult expulsions. Many clinicians schedule a FU visit after first menses or within 3 to 12 weeks following an IUD insertion, while others promote patientled IUD management, encouraging IUD-users to contact their clinician at any time if they have questions or concern about their IUD [2]. Hypothetically, increased surveillance of IUD position might increase the detection rate of IUD perforations [3]. However, there is contradictory evidence on what effect, if any, follow-up visits have the correct use of contraception and the detection of potential adverse events, such as perforations [4,5]. In 2003, a randomized controlled trial concluded that regular follow-up visits after IUD insertion did not increase the risk and side effects of IUD use [2].

The type of health system financing and method of healthcare professional (HCP) reimbursement is widely believed to influence clinical behaviour [6-8]. Europe has a heterogenous mix of health financing systems based on both tax-based and health insurance schemes. In addition, countries use a mix of fee-for-service, capitation and mixed payment systems to reimburse HCP. Although payment systems are often used to support policy objectives (e.g., cost containment, improved quality of care), the impact of these policies on specific health outcomes is often unclear [9]. Costs of contraception is known to influence contraceptive access $[10,11]$. Small increases to outof-pocket costs can significantly reduce demand [12]. While significant efforts have been made to understand drivers of contraceptive access, there is limited literature that explores how health care systems and payment methods influence the timing and methodology of contraceptive follow-up consultations.

Using data from the EURAS-IUD study, we conducted a retrospective analysis to examine whether routine IUD FU visit rates differed between European member states (Germany, Austria, Poland, Finland, Sweden and UK). Secondly, we assessed whether there is a trend between FU visits and IUD perforation rates in these six countries and if the country-specific primary care payment models might play a role in this pattern, if any.

\section{Material and methods}

EURAS-IUD (NCT00461175) was a multinational, prospective, non-interventional, long-term cohort study with recruitment in six

*Correspondence to: Pauline De Corte, Center for Epidemiology and Health Research Berlin, Invalidenstraße 115, 10115 Berlin, Germany, Tel: +4930 945101 ext 48; E-mail: p.de-corte@zeg-berlin.de

Key words: contraceptive IUD surveillance, perforation incidence, public health financing

Received: August 17, 2020; Accepted: September 04, 2020; Published: September 07, 2020 
European countries (Austria, Finland, Germany, Poland, Sweden and the UK) conducted between 2010 and 2016. Detailed methodology of EURAS-IUD is described elsewhere [13,14]. In short, the primary outcome of interest in EURAS-IUD was to measure the incidence and determine risk factors of uterine perforation. Recruitment of women was conducted via a network of health care professionals (HCPs) and based on local requirements. In Germany, Austria and Poland recruitment was predominantly through gynaecologists as these specialists inserted almost all IUDs. In the UK recruitment was extended to sexual health clinics which are staffed by General Practitioners (GPs), gynaecologists and midwives. In Sweden and Finland, recruitment was through both midwives and gynaecologists. All women with a newly inserted IUD (first ever or repeat/consecutive users) were eligible for enrolment. There were no explicit medical exclusion criteria.

At the time of IUD insertion, women completed a baseline questionnaire collecting information relating to medical and gynaecological history, age, body mass index, lifestyle factors (smoking, alcohol consumption, exercise, heavy lifting) and level of education. Baseline and 12-month follow-up data from all women recruited into EURAS-IUD before 31 July 2010 - in total 39,009 - formed the 12-60-month extension study cohort. Of these, all women with an IUD in situ at 12 months received a follow-up questionnaire 5 years after IUD insertion (30,144 women). Women who had their IUDs removed at 12 months due to perforation, expulsion or personal preference were not sent 5-year follow-up questionnaires, however, their data were included in the analyses of total incidence rates and risk factors for perforation. At each follow-up timepoint, women were asked specific questions related to potential IUD perforation, in addition to whether they had visited their HCP for a review of their IUD position and what procedures were performed to check the position (pelvic examination, transvaginal ultrasound). All potential adverse events were validated via direct contact with the patient or health care professional (HCP).

For this post-hoc analysis, we focused on the 12-60-month extension study cohort of 39,009 IUD users. The proportion of women who had a check-up examination, whether or not in combination with transvaginal ultrasound, were summarized per country cohort. Baseline characteristics (i.e., age and education), gynaecological history (i.e. first/ consecutive use, IUD-related complaints during first year of insertion and health professional who inserted the IUD) were summarized and compared between the different country cohorts.

We further evaluated the impact of primary health care financing on IUD check-up behaviour of both patients and HCPs. Additionally, the perforation incidence per 1,000 insertions stratified by country (with 95\% CI) was calculated and possible trends between check-up examinations and perforation incidence were evaluated. All events reported by the participating HCPs and/or participating women where any part of the device crossed the endometrium and entered the myometrium was considered a perforation. For the analysis, the most conservative approach was used to define perforation. Perforations confirmed via a diagnostic measure with high specificity (e.g., intraoperative, or endoscopic findings, imaging with unequivocal results: radiology, MRI, sonography; post-mortem) and/or confirmed by a health care professional supported by other evidence were included in the analysis.

All analyses were performed in SAS 9.1 (Cary, NC, USA). The EURAS-IUD study was approved by the ethical committee of the physician's association in Berlin, Germany, and the Ethics Committee of Hospital District of Southwest Finland. The other participating countries accepted these approvals.

\section{Results}

In total 39,009 participants formed the 12-60-month extension study cohort. Of these, all women with an IUD in situ at 12 months received a follow-up questionnaire 5 years after IUD insertion. Table 1 summarizes baseline characteristics and gynaecological data captured during follow-up in the population under study. No clear differences in age were seen between the different countries. More than half of the study population was aged between 30 and 49 years, ranging from $58.1 \%$ in Austria to $81.5 \%$ in Germany, 16.9 (Germany) to $28.8 \%$ (Sweden) was aged younger than 30 and 1.1 (Sweden) to 3.9\% (Finland and Poland) of women was older than 50. We observed different patterns in education between countries. In Finland, Austria, and Germany, respectively $49.6,65.6$ and $65.9 \%$ of women had no university entrance level, while for Poland, Sweden, and UK, respectively 83.2, 89.1 and $65.2 \%$ had a university or university entrance level. In Finland, Sweden, Poland, and UK half of women were first time users, while in Austria and Germany slightly more women were repeat or consecutive users (respectively 58.6 and 59.3\%).

IUDs were inserted primarily by a gynaecologist in Germany, Austria, and Poland. In the UK, $67.7 \%$ of women had their IUD inserted by the gynaecologist, $31.4 \%$ of women in specialized Contraception and Sexual Health $(\mathrm{CaSH})$ clinics by a trained Sexual Health physician or nurse and $0.90 \%$ by a midwife. In Sweden, only $10.8 \%$ of women had their IUD inserted by a gynaecologist while $89.2 \%$ of women had their IUD inserted by a midwife.

We also summarized the number of women who self-reported a problem with the inserted IUD within the first year of the insertion and if she visited a physician due to this reported problem. In Poland only $9.1 \%$ reported a problem with her IUD, of which $70.2 \%$ visited a physician for this complaint. In Germany, Finland, and UK a comparable number of women resp. $13.5 \%, 14.6 \%$ and $16.0 \%$ reported a problem and respectively $61.8 \%, 58.3 \%$ and $78.7 \%$ of those visited an HCP. A higher number of women reported an IUD problem in Austria (19.1\%) and Sweden (20.0\%) of which respectively $69.0 \%$ and $48.5 \%$ visited a physician because of this reported problem.

Differences in routine check-up IUD-care procedures were seen for the different European countries. The proportion of women reporting a medical FU visit and IUD position check varied per country ranging from $15.5 \%$ in Finland to $90.4 \%$ in Austria (Table 2). Whether a woman had a transvaginal ultrasound also depended on local HCP practice. In Germany, Austria, and Poland most women had a follow-up visit (76.3\%, $90.4 \%$ and $78.4 \%$, respectively). Most of these women also had a transvaginal ultrasound to confirm the IUD position $(85.6 \%, 90.5 \%$ and $86.2 \%$, respectively). In contrast, although $58.6 \%$ of women in the United Kingdom were examined, only $19.3 \%$ of these women went on to having a transvaginal ultrasound. The opposite pattern was seen in Finland and Sweden, where a relatively small number of the total study population was examined (respectively $15.5 \%$ and $33.6 \%$ had a FU visit), however, those who had a physical check of their IUD position were more likely to also have a transvaginal ultrasound (respectively $62.6 \%$ and $51.0 \%$ ).

As presented in Table 3, the perforation incidence per 1,000 insertions was in general low, varying between 0.32 in Austrian and 3.20 in UK. We observed no correlation between check-up rates and IUD perforation detection $\left(\mathrm{R}^{2}=0.07\right)$. In Germany and Austria, where check-up rates are in general high, the perforation incidence proportion was 1.91, (95\%CI 1.29-2.72) and 0.32, (95\%CI 0.04-1.17), respectively. This compares to perforation incidence proportions of 0.94 (95\%CI 0.11-3.39), 3.20 (95\%CI 2.25-4.41) and 2.09 (95\%CI 0.77- 
Table 1. Overview of baseline characteristics and gynaecological data captured during follow-up.

\begin{tabular}{|c|c|c|c|c|c|c|c|}
\hline & All & Germany & Austria & UK & Finland & Poland & Sweden \\
\hline & \multicolumn{7}{|c|}{$\mathrm{N}$ of women $(\%)$} \\
\hline \multicolumn{8}{|l|}{ Age (in years) } \\
\hline$<20$ & $485(1.2 \%)$ & $112(0.7 \%)$ & $90(1.5 \%)$ & $204(1.8 \%)$ & $35(1.2 \%)$ & $2(0.4 \%)$ & $42(2.0 \%)$ \\
\hline $20-29$ & $7,904(20.3 \%)$ & $2,546(16.2 \%)$ & $1,135(18.3 \%)$ & $2,834(24.5 \%)$ & $717(25.0 \%)$ & $101(19.5 \%)$ & $571(26.8 \%)$ \\
\hline $30-39$ & $16,025(41.1 \%)$ & $6,566(41.7 \%)$ & $2,565(41.4 \%)$ & $4,546(39.3 \%)$ & $1,186(41.4 \%)$ & $261(50.4 \%)$ & $901(42.3 \%)$ \\
\hline $40-49$ & $13,788(35.3 \%)$ & $6,272(39.8 \%)$ & $2,303(16.7 \%)$ & $3,670(31.7 \%)$ & $816(28.5 \%)$ & $134(25.9 \%)$ & $593(27.8 \%)$ \\
\hline $50+$ & $807(2.1 \%)$ & $245(1.6 \%)$ & $100(1.6 \%)$ & $307(2.7 \%)$ & $111(3.9 \%)$ & $20(3.9 \%)$ & $24(1.1 \%)$ \\
\hline \multicolumn{8}{|l|}{ Education } \\
\hline No university entrance level & $19,818(51.9 \%)$ & $10,368(65.9 \%)$ & $4,061(65.6 \%)$ & $3,760(32.5 \%)$ & $1,421(49.6 \%)$ & $84(16.2 \%)$ & $124(5.8 \%)$ \\
\hline University entrance level & $8,546(22.4 \%)$ & $1,963(12.5 \%)$ & $1,221(19.7 \%)$ & $3,373(29.2 \%)$ & $755(26.4 \%)$ & $174(33.6 \%)$ & $1,060(49.7 \%)$ \\
\hline University & $9,838(25.7 \%)$ & $3,102(19.7 \%)$ & $784(12.7 \%)$ & $4,166(36.0 \%)$ & $689(24.0 \%)$ & $257(49.6 \%)$ & $840(39.4 \%)$ \\
\hline \multicolumn{8}{|l|}{ HCP specialization } \\
\hline Midwife & $2,193(5.6 \%)$ & $0(0.0 \%)$ & $0(0.0 \%)$ & $106(0.9 \%)$ & $184(6.4 \%)$ & $2(0.4 \%)$ & $1,901(89.2 \%)$ \\
\hline Gynecologist & $33,191(85.1 \%)$ & $15,741(100.0 \%)$ & $6,193(100.0 \%)$ & $7,830(67.7 \%)$ & $2,681(93.6 \%)$ & $516(99.6 \%)$ & $230(10.8 \%)$ \\
\hline IUD clinics & $3,624(9.3 \%)$ & $0(0.0 \%)$ & $0(0.0 \%)$ & $3,624(31.3 \%)$ & $0(0.0 \%)$ & $0(0.0 \%)$ & $0(0.0 \%)$ \\
\hline \multicolumn{8}{|l|}{ IUD use } \\
\hline First time & $18,320(47.0 \%)$ & $6,481(41.2 \%)$ & $2,575(41.6 \%)$ & $6,453(55.8 \%)$ & $1,514(52.8 \%)$ & $277(53.5 \%)$ & $1,020(47.9 \%)$ \\
\hline Repeat/consecutive & $20,689(53.0 \%)$ & $9,260(58.8 \%)$ & $3,618(58.4 \%)$ & $5,108(44.2 \%)$ & $1,351(47.2 \%)$ & $241(46.5 \%)$ & $1,111(52.1 \%)$ \\
\hline \multicolumn{8}{|l|}{ IUD reported problems } \\
\hline Reported problem with IUD & $6,058(59.8 \%)$ & $2,130(13.5 \%)$ & $1,182(19.1 \%)$ & $1,854(16.0 \%)$ & $418(14.6 \%)$ & $47(9.1 \%)$ & $427(20.0 \%)$ \\
\hline Of which, visited the physician because of this & $4,076(40.2 \%)$ & $1,317(61.8 \%)$ & $816(69.0 \%)$ & $1,460(78.7 \%)$ & $243(58.3 \%)$ & $33(70.2 \%)$ & $207(48.5 \%)$ \\
\hline
\end{tabular}

Note: missing values are not represented in this table

Table 2. Women with a reported check-up of IUD position, stratified by country.

\begin{tabular}{|c|c|c|c|}
\hline & $\mathbf{n}^{*}$ & $\%$ with check-up after IUD insertion & $\begin{array}{l}\% \text { with transvaginal ultrasound (of those } \\
\text { with check-up) }\end{array}$ \\
\hline Austria & 6,193 & 90.4 & 90.5 \\
\hline Germany & 15,741 & 76.3 & 85.6 \\
\hline Poland & 518 & 78.4 & 86.2 \\
\hline Sweden & 2,131 & 33.6 & 51.0 \\
\hline Finland & 2,865 & 15.5 & 62.6 \\
\hline United Kingdom & 11,561 & 58.6 & 19.3 \\
\hline Total & 39,009 & 66.5 & 68.0 \\
\hline
\end{tabular}

$*_{n}=$ number of women with a newly inserted IUD (both first and repeat/consecutive users).

Table 3. Perforation incidence per 1,000 insertions stratified by country $(95 \% \mathrm{CI})$.

\begin{tabular}{|c|c|c|c|}
\hline & Number of perforations & Incidence proportion** & 0.32 \\
\hline Austria & 2 & 1.91 & $0.04-1.17$ \\
\hline Germany & 30 & -- & $1.29-2.72$ \\
\hline Poland & 0 & -- & 0.94 \\
\hline Sweden & 2 & 2.09 & \\
\hline Finland & 6 & 3.20 & $0.11-3.39$ \\
\hline United Kingdom & 37 & 1.97 & $2.25-4.41$ \\
\hline Total & 77 & $1.56-2.47$ & \\
\hline
\end{tabular}

**Incidence proportion: Perforation Incidence per 1,000 insertions.

4.55) in Sweden, UK, and Finland, where less women attended routine check-up examinations. No perforations were reported in the Polish study population and therefore, trends between routine check-up and perforation incidence could not be assessed. Figure 1 shows the correlation between women with an IUD check-up and perforation incidence stratified by country.

We additionally checked if there is a potential trend between the observed healthcare data and the main source of primary health care financing. Table 4 presents the main source of health care financing per country. Based on this table we conclude a possible trend in behaviour of HCP practice coming from countries that rely largely on social health insurance (SHI) system and countries where health expenditure is mainly operated by a taxation-based system. In countries with a SHI system for primary health care coverage - Germany, Austria, and Poland - we observed in general higher check-up rates compared to countries with a taxation-based system - Sweden, Finland and UK.

\section{Implications for practice and/or policy}

Our study provides preliminary insight in global variability in patient-health care seeking behavior in relation to IUD use. Our findings suggest that the primary health care payment model might play an important role in patient-decision making and IUD surveillance while stressing that more research is needed to understand if and how health care financing might affect a potential trend. 


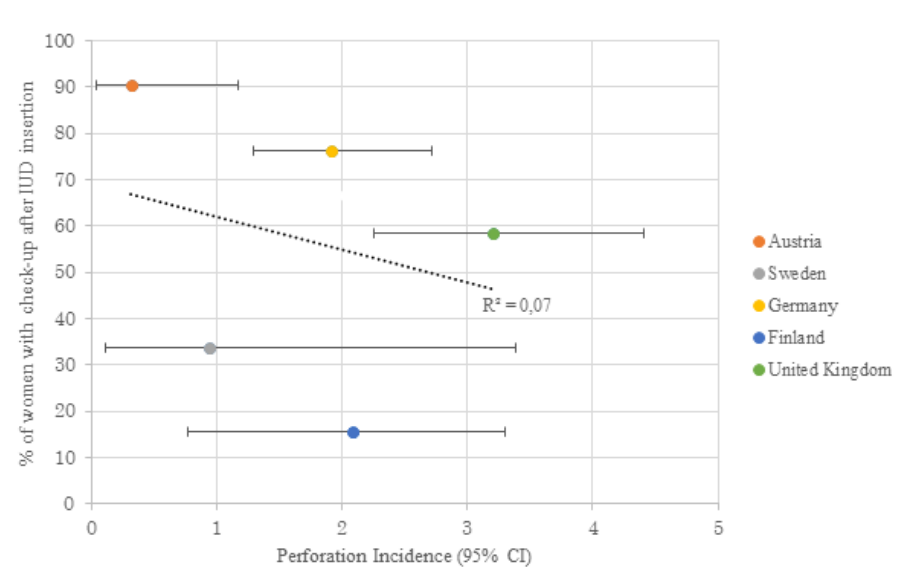

Figure 1. Percentage of women with a check-up and perforation incidence stratified per country.

\section{Discussion}

To our knowledge, this is the first study comparing routine IUD checks following IUD insertion and the incidence of IUD perforation in clinical practice across several Western European countries. We identified a noticeable difference in check-up rates between the different countries included in this study. Based on the collected baseline and gynaecological characteristics, we examined plausible explanations for these obtained differences in routine IUD check-up rates of the women. When evaluating age, education, first/consecutive use, IUDrelated complaints during first year of insertion and medical person who inserted the IUD, we could not conclude a possible clear trend between these variables and check-up behaviour.

In addition, we observed no clear trend in check-up rates and perforation incidences $\left(R^{2}=0.07\right)$. Perforation incidence proportions did not correspond in analogous manner to check-up patterns. Countries in which check-up rates were higher did not necessarily correspond with a higher perforation incidence and vice versa.

On the other hand, we hypothesize that a possible correlation might exist between health system financing and IUD check-up rates. In our study, countries health system financing could be broadly categorised into either social health insurance (SHI) schemes or taxation-based systems. We noted a similar trend in check-up rates in countries with healthcare payment based on a SHI - Austria, Germany, and Poland and those with a taxation-based healthcare payment model - Finland, Sweden and the UK.

The type of healthcare systems financing within a country is known to influence physician behaviour and patient response and might explain differences in healthcare seeking behaviour seen in our study [15].

Ellis et al. [16] described that under tax-financed systems, health care providers may end up under-serving patients due to budgetary restraints. In Finland and Sweden, both tax-based health financed system, we observed low levels of IUD post-insertion appointments. This result might suggest a trend towards the under-servicing of IUD users in these countries. However, if true, we would also expect to observe more women in Finland and Sweden complaining of problems with their IUD and/or a difference in IUD perforation rates. Our data showed no such differences.

In Germany, SHI-accredited physicians in outpatient care are generally reimbursed on a fee-for-service (FFS) basis according to a uniform fee schedule negotiated between sickness funds and physicians [17]. Within the primary health care payment model, these physicians receive an additional fee if a transvaginal ultrasound was used to confirm the IUD position. Reimbursement for this transvaginal ultrasound is reflected in the EURAS-IUD data; almost all women in Germany received a transvaginal ultrasound as an additional procedure during a routine IUD check. However, more frequent IUD surveillance, including transvaginal ultrasound, did not necessarily result in an increased detection rate of IUD perforations. We observed no correlation between IUD perforation rates and increased IUD surveillance. We hypothesise that SHI health financing, in combination with fee-for-service payment models, may lead to unnecessary servicing of IUD users.

In contrast to both the Scandinavian and Central European countries, a third model of care was seen in the UK, where of those women with a check-up (58.6\%), only $20 \%$ were examined with transvaginal ultrasound. A plausible explanation for this difference could be that in the UK access to specialist services, including ultrasound, is generally possible only after referral from a General Practitioner (GP). Routine check of an IUD position it likely to form part of a GP checkup, however, the physical- and time-related distance of separating the IUD-examiner from the sonographer may have limited provision of transvaginal ultrasounds to women with 'difficult' or 'problematic' IUDs (i.e., where the IUD threads could not be visualised).

The EURAS-IUD design has proven to contribute to the validity of results by combining several methodological strengths such as (1) comprehensive follow-up procedures and very low loss to follow-up to minimize underreporting; (2) a study population representative for IUD users under routine clinical conditions; (3) validation of outcomes of interest and (4) supervision by an independent Safety Monitoring and Advisory Council as well as scientific independence from the study funder. In addition to these methodological strengths, special attention was paid to typical biases, which are described elsewhere $[13,14]$. This study also provided a large sample size in six European countries and thus enables the comparison of check-up rates and IUD perforation incidence in routine care after IUD insertion. Efforts were made to include both large and small European countries with differing regional/rural populations reflecting the general population of European IUD users.

However, this study has also methodological limitations. In this post-hoc descriptive study, data was collected for other research purposes as used for this secondary analysis, meaning that important variables and information regarding health care could not be obtained from the women. Proportions were not compared using formal statistical testing due to concerns regarding multiple testing. Therefore, the study remains of exploratory nature. More empirical research is necessary to assess the relationship between cofactors such as HCP experience or IUD problems and perforation.

It is also important to recognise that the health system and payment method (e.g., fee-for-service, salary, capitation) is only one factor that affects contraceptive decisions. The existence of a gatekeeper, funding for contraception, differences in how IUD services are funded, recommendation for performing self-checks, patients' expenditure for and access to health care and the cultural milieu in which these operate should also be considered. In Austria and Germany, for example, women must pay for the insertion of the device, but a free-of-charge ultrasound to check if the IUD is still in place is included within the first 4 to 6 weeks after IUD insertion for Austria, and within the first year of IUD insertion for Germany. These factors may help to explain 
the higher check-up rates for these countries. Moreover, the willingness of women to perform a self-check after IUD placement may influence HCP follow-up recommendations. Inter- and intra-country differences in the advice given to women to perform a self-check after IUD insertion exist [1] but were not assessed during the study.

Additionally, in practice, most financing systems are not solely based on one method but follow a mixed-methods approach reflecting political and economic history. Because of its complexity, the behaviour of a health-care system is not easy to predict. Depending on the country, the cost of the IUD and the placement might be charged to the health care system, insurance companies, non-profit organizations or to the women themselves [3].

Lastly, since 2010 the IUD landscape has changed, with the introduction of LNG containing IUDs of different strengths. We do not believe that these changes would have affected the interpretation on our results. We assume that the same women are likely to choose an IUD as their contraceptive method. To our knowledge, there have been no changes in access to reproductive health technologies and no significant changes in healthcare payment structures in the study countries.

\section{Conclusion}

There was a noticeable difference in check-up rates in routine care after IUD insertion between different European countries. Increased IUD surveillance did not correlate with increased detection of IUD perforations. We speculated on the possible impact of the principal system of health financing, routine IUD surveillance and perforation incidences. More research is needed to understand the influence of health-system financing, HCP reimbursement and downstream clinical outcomes.

\section{Funding statement}

The EURAS-IUD study was a post authorization safety study funded with an unconditional grant from Bayer Schering pharm. The study was supervised by an independent Safety Monitoring and Advisory Council with full authority over the study. The funder had no access to the source data and did not participate in designing the study or analyzing the data.

\section{Acknowledgments}

The authors would like to express their appreciation to the members of the independent Safety Monitoring and Advisory Council for their constructive criticism and unfailingly fair scientific discussion. The authors would also like to highlight the contributions of numerous colleagues who were responsible for the field work in the individual countries. They clarified data inconsistencies and missing data, validated patient-reported adverse events with patience, care and tenacity, and their untiring commitment enabled a remarkably low loss to follow-up rate.

\section{References}

1. Buhling KJ, Zite NB, Lotke P, Black K (2014) Worldwide use of intrauterine contraception: a review. Contraception 89: 162-173. [Crossref]

2. Neuteboom K, de Kroon CD, Dersjant-Roorda M, Jansen FW (2003) Follow-up visits after IUD-insertion: sense or nonsense? A technology assessment study to analyze the effectiveness of follow-up visits after IUD insertion. Contraception 68: 101-104. [Crossref]

3. ACOG (1993) The intrauterine device. ACOG technical bulletin number 164--February 1992 (replaces no. 104, May 1987). Int J Gynaecol Obstet 41: 189-193. [Crossref]

4. Steenland MW, Zapata LB, Brahmi D, Marchbanks PA, Curtis KM (2013) The effect of follow-up visits or contacts after contraceptive initiation on method continuation and correct use. Contraception 87: 625-630. [Crossref]

5. Draper IB, Haque MS, McManus RJ (2012) Routine intrauterine device checks: are they advisable? J Fam Plann Reprod Health Care 38: 15-18. [Crossref]

6. Cashin Ch, Chi YL, Borowitz M, Smith P, Thomson S (2014) Paying for Performance in Health Care: Implications for Health System Performance and Accountability. Available from: https://www.euro.who.int/en/publications/abstracts/paying-for-performance-inhealth-care.-implications-for-health-system-performance-and-accountability-2014

7. Dudley RA, Miller RH, Korenbrot TY, Luft HS (1998) The impact of financial incentives on quality of health care. Milbank $Q 76: 649686$. [Crossref]

8. Conrad D, Christianson J (2004) Penetrating the 'black box': financial incentives for enhancing the quality of physician services. Med Care Res Rev 61: 37S-68S. [Crossref]

9. Gosden T, Forland F, Kristiansen I, Sutton M, Leese B, et al. (2001) Impact of payment method on behaviour of primary care physicians: A systematic review. J Health Serv Res Policy 6: 44-55. [Crossref]

10. Gariepy AM, Simon EJ, Patel DA, Creinin MD, Schwarz EB (2011) The impact of out-of-pocket expense on IUD utilization among women with private insurance. Contraception 84: e39-e42. [Crossref]

11. Frost JJ and Darroch JE (2008) Factors associated with contraceptive choice and inconsistent method use. Perspect Sex Reprod Health 40: 94104. [Crossref]

12. Gill J, Taylor D (2017) Improving Access to Contraception. London School of Economics and Political Science. Available from: https://www.lse.ac.uk/business-andconsultancy/consulting/consulting-reports/improving-access-to-contraception

13. Heinemann K, Reed S, Moehner S, Do Minh T (2015) Risk of uterine perforation with levonorgestrel-releasing and copper intrauterine devices in the European Active Surveillance Study on Intrauterine Devices. Contraception 91: 274-279. [Crossref]

14. Heinemann K, Reed S, Moehner S, Do Minh T (2015) Comparative contraceptive effectiveness of levonorgestrel-releasing and copper intrauterine devices: the European Active Surveillance Study for Intrauterine Devices. Contraception 91: 280-283. [Crossref]

15. Wagstaff A (2009) Social health insurance vs. tax-financed health systems - evidence from the OECD (English). Policy Research working paper; no. WPS 4821. World Bank Group. Washington, DC, USA. Available from: http://documents.worldbank. org/curated/en/545121468028868365/Social-health-insurance-vs-tax-financed-healthsystems-evidence-from-the-OECD

16. Ellis RP, Miller M (2008) Provider Payment Methods and Incentives. International Encyclopedia of Public Health. H. Kris. Oxford, Academic Press. pp: 395-402.

17. Blümel M and Busse R. Germany: International Health Care System Profiles. 2020 [online]. Available from: https://international.commonwealthfund.org/countries/ germany/

Copyright: (C2020 Pauline De Corte. This is an open-access article distributed under the terms of the Creative Commons Attribution License, which permits unrestricted use, distribution, and reproduction in any medium, provided the original author and source are credited. 